\title{
Will the COVID-19 pandemic accelerate the implementation of single-day coronary angioplasty in Poland?
}

\author{
Andrzej Ciszewski \\ Department of Cardiology and Invasive Cardiology, National Institute of Cardiology, Warsaw, Poland
}

Adv Interv Cardiol 2020; 16, 2 (60): 184-186 DOI: https://doi.org/10.5114/aic.2020.95852

\section{Introduction}

Single-day coronary angioplasty (S-D PCl) is becoming a preferred approach to percutaneous coronary intervention (PCI) among physicians and patients in developed countries [1-3]. Its safety has been proved in low and intermediate risk patients by randomized and observational studies [4-6].

The recent COVID-19 pandemic exerts extraordinary pressure to reduce the risk of infection transmission. In such a critical moment S-D PCI has emerged as a leader in preventing the spread of in-hospital infections.

\section{Case report and results}

I present a case of a 65-year-old male patient who, due to accelerated typical angina symptoms, could not wait for the decline of the pandemic and had right coronary artery (RCA) PCl performed in a single-day coronary invasive unit (S-D unit) on March 25 th, 2020 . Figure 1 shows the "culprit lesion": diffused, long RCA stenosis: before and after implantation of 2 drug-eluting stents (DES). Blood tests, routinely necessary to be performed before an invasive procedure, had been evaluated 3 weeks earlier by a family doctor.

Now, in the COVID-19 era, the patient was subjected to special precautions and a protocol introduced in our hospital to diminish any risk for infection transmission. First there was a short epidemiological interview concerning the suspicion of COVID-19 infection, body temperature measurement and rapid blood test "2019 nCoV IgG/IgM", Vazyme Biotech Co. Ltd. After a negative result (10 $\mathrm{min}$ ) and admission to hospital, the patient wore a face mask until his discharge and was placed in a single room in an S-D unit or in a cath lab. Special at- tention was paid so that during an about 6-hour-long stay in the S-D unit only one dedicated nurse and one doctor were in personal contact with the patient.

The personnel performing the invasive procedure in the cath lab were additionally equipped with full face plastic shields along with routinely used face masks, caps and gloves.

The crucial results and data concerning the risk for COVID-19 transmission are as follows. Length of stay in hospital 7 h 30 min (admission 7:05, discharge 14:35). The number of personnel with contact defined as dangerous for COVID-19 transmission (distance $<2 \mathrm{~m}$, duration $>3$ min) was limited to 5 persons. One person admission formalities and epidemiological examination, 2 people (1 nurse and 1 treating/invasive cardiologist) in an S-D invasive unit, 2 people in a cath lab (1 technician and 1 nurse). The invasive procedure was performed by the same invasive cardiologist who was the treating physician. All hospital personnel were obliged to wear masks and gloves during any contact with the patient.

After a successful PCI procedure, the patient stayed in a single room at the S-D unit in a comfortable armchair, received i.v. $1000 \mathrm{ml}$ of fluid and his heart rhythm and vital functions were monitored by a heart monitor. The patient was discharged in stable clinical condition, without any chest discomfort or ECG ischemic changes, after radial compression removal and resuming some physical activity. Our patient selection and management protocol for S-D PCl was precisely described in a previous publication [7]. In brief: we accept patients without age limit, possible radial approach, ejection fraction (EF) > 30\%, normal renal function, domicile not far from hospital (transport < $60 \mathrm{~min}$ ) and no planned $\mathrm{PCl}$ of: left main, coronary bypass and chronic total occlusion.

\section{Corresponding author:}

Andrzej Ciszewski MD, PhD, Department of Cardiology and Invasive Angiology, National Institute of Cardiology, 42 Alpejska St, 04-628 Warsaw, Poland, phone: +48 22 3434267, e-mail: aciszewski@ikard.pl

Received: 2.04.2020, accepted: 11.05.2020. 

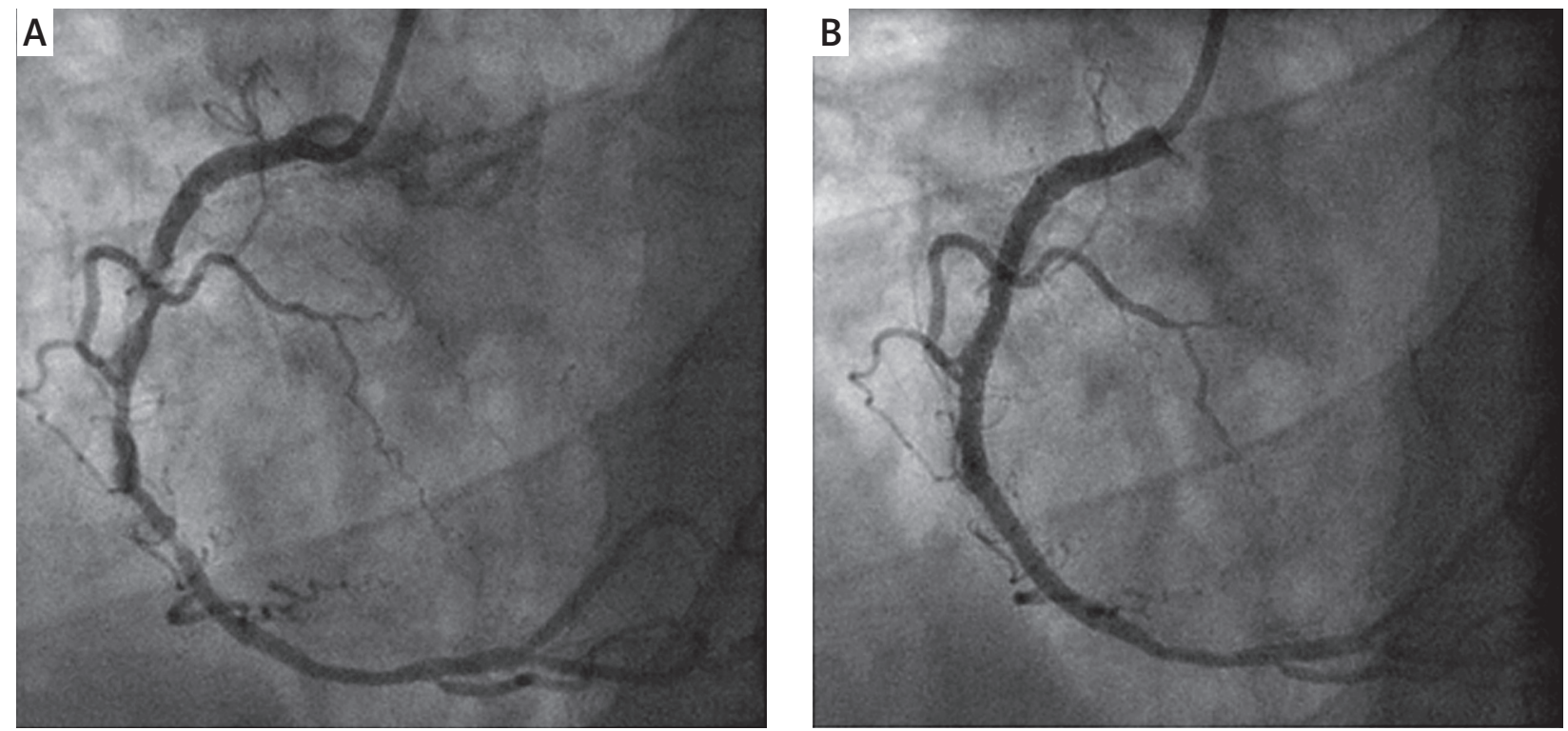

Figure 1. Right coronary artery: A - diffused, long stenosis, B - artery after 2 Des Stents implantation $(3.0 \times 28 \mathrm{~mm}$, $2.75 \times 23 \mathrm{~mm}, 14 \mathrm{~atm})$

\section{Discussion}

Numerous studies have shown that early PCI complications, if they do occur, reveal themselves during the first $6 \mathrm{~h}$ after the procedure, and the period between 6 and $24 \mathrm{~h}$ is almost free of unexpected events [4-6]. In the majority of hospitals in Poland, a planned $\mathrm{PCl}$ procedure is associated with about $48 \mathrm{~h}$ long hospitalization and a patient is exposed to contact with at least 3-4 shifts of personnel and with other hospitalized patients. Despite the pressure from the authorities to stop planned hospitalizations and procedures, the spectrum of patients with coronary heart disease is complex and they cannot be divided into two groups: an emergency (ACS) group, and a stable angina group which can wait until the end of the pandemic. There exists an important group of patients, like our case, with accelerated symptomatic angina who should undergo urgent invasive diagnosis and treatment in a short (1-2 weeks) time because of a risk of myocardial infarction and death. The development and propagation of S-D PCI seem the best solution for these patients.

In the era of the COVID-19 pandemic and a significant reduction of invasive procedures, we should concentrate on two aims: identification of patients "in danger" on our waiting lists and reduction of risk of infection transmission from, or to a patient, during hospital stay. The steps introduced in our hospital seem to be well balanced between the continuation of necessary cardiological treatment and the risk of exposure to a potentially life-threatening infection. First there was telephone verification of our waiting list by an experienced medical secretary, occasionally supported by a cardiologist. Nowadays, many patients with stable, mild symptoms definitely prefer to have invasive diagnostics delayed.
The identification of patients with severe or accelerated angina symptoms who accept short hospitalization and medical contact for invasive cardiology despite the COVID-19 pandemic is performed more easily than we expected. During the last weeks of our activity the percentage of patients qualified after coronary angiography for "ad hoc" or planned angioplasty increased to > 50\%. S-D PCl development and propagation seem the best solution for $\mathrm{PCl}$. The special precautions introduced recently in our institution because of the pandemic were described in the Case Presentation section. They are aimed at protecting both patients and personnel. The most important elements are: epidemiological interview and examination of a patient regarding the suspicion of COVID-19 infection at admission, stay in a single room, obligation for a patient and personnel to wear face masks and gloves during contact, and provision of full face plastic shields for cath lab personnel. As in our case it is important to define at admission which personnel members are assigned to contact and take care of each individual patient to reduce unnecessary exposure. The causes of delayed implementation of S-D PCI in Poland are complex. However, the recent COVID-19 pandemic should facilitate overcoming the existing organizational and psychological barriers. It would be very helpful if the National Health System (NFZ) in agreement with the Association of Cardiovascular Interventions could announce that the development of S-D PCl procedures in Poland will not cause a reduction of the reimbursement of $\mathrm{PCl}$ procedures.

\section{Conflict of interest}

The author declares no conflict of interest. 


\section{References}

1. Taxiarchi P, Kontopantelis E, Martin GP, et al. Same-day discharge after elective percutaneous coronary intervention. Insight from the British Cardiovascular Intervention Society. JACC CardiovasC Interv 2019; 12: 1479-94.

2. Amin AP, Pinto D, House JA, et al. Association of same-day discharge after elective percutaneous coronary intervention in the United States with costs and outcomes. JAMA Cardiol 2018; 3 : 1041-9.

3. Kwok CS, Sunil VR, Gilchrist IC, et al. Relation of lenght of stay to unplanned readmission for patients who undergo elective percutaneous coronary intervention. Am J Cardiol 2019; 123: 33-43.

4. Slagboom T, Kiemeneji F, Laarman GJ, et al. Actual outpatient PTCA: results of the OUTCLAS pilot study. Catheter Cardivasc Interv 2001; 53: 204-8.

5. Hayde GS, Koch KT, de Winter RJ, et al. Randomized trial comparing same-day discharge with overnight hospital stay after percutaneous coronary intervention: results of the Elecive $\mathrm{PCl}$ in Outpatient Study (EPOS). Circulation 2007; 115: 2299-306.

6. Cordoba-Soriano JG, Jimenez-Mazuecos J, Rivera-Juarez A, et al. Safety and feasibility of outpatient percutaneous coronary intervention in selected patients: a Spanish Multicenter Registry. Rev Esp Cardiol 2017; 70: 535-42.

7. Ciszewski A. One-day coronary angioplasty. Patients' characteristics and results of 30-day. Kardiol Pol 2020, 19 ${ }^{\text {th }}$ Feb, DOI: 10.33963/KP.15196. 